DOI: https://doi.org/10.29105/gmjmx17.33-6

Artículos

\title{
JÓVENES Y PUBLICIDAD: LAS IMPLICACIONES DE LA COMUNICACIÓN BOCA A BOCA
}

\section{YOUTH AND ADVERTISING: THE IMPLICATIONS OF WORD OF MOUTH COMMUNICATION}

\section{María Elisa Sabre}

Universidad Siglo 21, Argentina

ORCID: https://orcid.org/0000-0001-9040-3281

Autor para correspondencia: María Elisa Sabre, email: elisa.sabre@ues21.edu.ar

\section{Resumen}

Este artículo resume algunos resultados de un estudio exploratorio descriptivo, de carácter cuantitativo, que tuvo como objetivo analizar las variables relacionadas con el consumo y decisiones de compra de los jóvenes argentinos y analizar el rol de las diferentes formas de publicidad en esos procesos. A través de un cuestionario autoadministrado, se recogió información sobre confianza y motivación frente a diferentes formatos publicitarios, así como variables relativas a hábitos de consumo. Los resultados demuestran que las opiniones difundidas boca a boca son la fuente de información y el canal de recomendación más fiable y motivador para la compra en los jóvenes. Pero la efectividad del boca a boca no se limita al mundo offline, sino que se extiende al ámbito de Internet en el llamado boca a boca electrónico. En este sentido, es más probable que los consumidores confien en los consejos y opiniones de sus pares (aunque sean personas desconocidas) que en los anuncios que provienen directamente de la marca a través de la publicidad tradicional a la hora de tomar una decisión de compra. Esto también se aplica a cualquier consejo previo a la compra e información sobre los productos que se obtiene a través de dichas interacciones.

Palabras claves: publicidad, boca a boca, boca a boca electrónico, jóvenes, decisiones de compra.

\begin{abstract}
This paper summarizes some results of a descriptive, quantitative exploratory study aimed to analyze variables related to purchasing decisions of young Argentines and to analyze the role of several forms of advertising in these processes. Through a self-administered questionnaire, information on trust and motivation of different advertising formats was collected, as well as variables related to consumption habits. The results show that word of mouth is the most reliable and motivating source of information for the young consumers. But its effectiveness is
\end{abstract}

Global Media Journal México 17(33). julio - diciembre 2020. Pp. 123-138. 
not limited to the offline world, but extends to the Internet in the so-called electronic word of mouth. Consumers are more likely to rely upon the advice and opinions of their peers (even strangers) than in the ads that come directly from the brand through traditional advertising when making a buying decision. This also applies to any pre-purchase advice and brand information which is obtained through such interactions.

Keywords: advertising, word of mouth, electronic word of mouth, youth, buying decisions.

Recibido: 22/10/2020

Aceptado: $27 / 11 / 2020$

\section{Introducción}

Las nuevas tecnologías de la información y la comunicación están provocando profundos cambios y transformaciones de naturaleza social, cultural y económica al punto de que incluso se considera que estamos transcurriendo un nuevo período en la civilización humana conocido como 'sociedad de la información y del conocimiento'.

Esto quiere decir que los cambios que se originan a partir de las TIC están configurando una nueva sociedad. Una sociedad donde hay un mayor acceso a la educación y a la información, donde existen medios interactivos que privilegian el acceso individual sobre el masivo, donde se multiplica constantemente el volumen de información disponible y donde la permanente conectividad y las comunidades virtuales producen cambios en las relaciones interpersonales y donde surgen nuevas formas de trabajo y de ocio (Vivas López, 2007).

Como no podía ser de otra manera, los escenarios sociales donde los medios de comunicación y los grupos sociales intervienen e interactúan también han cambiado: ya no hay direccionalidad desde un emisor a un receptor, sino complejos procesos de interacción donde los grupos sociales y los propios sujetos individuales tienen un progresivo protagonismo (Benavides Delgado, 2012) y donde existe una dilución de las fronteras que tradicionalmente han separado los distintos tipos de medios (AIMC, 2010).

En este contexto, el panorama publicitario también está evolucionando a un ritmo extraordinario ya que la proliferación de medios y los avances tecnológicos crean nuevas formas conexión con los diferentes públicos. Junto al poder publicitario de argumentar, de motivar y de persuadir, se suma un nuevo poder: el de la telepresencia, al que finalmente se incorpora Internet y el teléfono móvil como 
nuevos media. Internet encarna una revolución en las comunicaciones inédita hasta el momento, donde la interactividad grana protagonismo frente a los medios masivos, por naturaleza unidireccionales. Los consumidores son, por primera vez y al mismo tiempo, productor, emisor y receptor (Costa, 2007, p. 53).

\section{Marco teórico}

Todas estas nociones han dado paso a un nuevo ideal de consumidor, que ya no puede ser caracterizado como un ser manipulado, que compra de manera impulsiva ante la avalancha de mensajes que lo rodean. El consumidor actual puede ser definido como un

ser más crítico y consciente de su poder y de su impacto a partir, no sólo de sus decisiones de compra, sino de su influencia en la compra de los otros y en la reputación de las organizaciones, un actor que quiere asumir un rol más participativo en los procesos de consumo (Mejia-Giraldo, 2016, p. 188).

La generación nacida después de 1980 se caracteriza, por encima de todo, por hacer uso de estas nuevas tecnologías de la información y comunicación desde la infancia. Este grupo, que se conoció en una primera instancia como 'Generación Y', poco a poco se ha hecho popular a través de un concepto acuñado por Howe y Strauss (2000) y que hace referencia a que es la primera generación que alcanza la mayoría de edad en el nuevo milenio: los millennials. Esta generación puede ser considerada como la primera en utilizar los medios digitales más que los tradicionales (Geraci y Nagy, 2004) y es una de las que más está en el punto de mira de los anunciantes en las últimas décadas debido al creciente potencial de consumo que alberga y su destacado papel como prescriptora e influenciadora en los procesos de compra.

La gran cultura publicitaria que tienen estos jóvenes los convierte en públicos objetivo altamente informados $\mathrm{y}$ mucho más críticos con los mensajes publicitarios, a diferencia de lo que sucedía en las generaciones precedentes. $\mathrm{La}$ juventud actual valora en la publicidad su dimensión informativa como pieza clave de una sociedad de consumo, pero, sin duda, es menos crédula ante la dimensión persuasiva de esos mensajes (Muela-Molina y Pazos, 2010).

Algunos autores consideran que la razón por la cual los jóvenes relativizan la influencia de la publicidad tradicional en sus decisiones de consumo es porque son cada vez más conscientes de la importancia de la "publicidad que se ejerce entre iguales" (Sánchez et al., 2004, p. 120). Los jóvenes son además los principales usuarios de los medios surgidos gracias a las nuevas tecnologías, gracias a los cuales cualquier individuo tiene la capacidad de hacer llegar 
sus opiniones acerca de marcas, productos y empresas al resto de la sociedad, y estos mensajes conviven en el ciberespacio con aquellos planificados y controlados por los anunciantes (Muela-Molina y Pazos, 2010).

Es así que los espacios de comunicación convierten en "espacios de conversación" donde los consumidores participan activamente en la coconstrucción de la imagen de una marca generando mensajes que influyen en la imagen de marca que generan otros consumidores y que resultan determinantes en los procesos de toma de decisión. La relación entre la publicidad y los consumidores se ha instalado en un "un diálogo permanente, mutante, que se retroalimenta y adapta a los vaivenes de un entorno digital simultáneamente construido por todos los participantes" (Tur-Viñes, 2016, p. 2).

\section{Publicidad boca a boca}

El boca a boca [word of mouth $\{\mathrm{WOM}\}]$ puede ser definido como una "comunicación cara a cara sobre una marca, producto o servicio entre personas que se supone no tienen conexión con una entidad comercial" (Arndt, 1967, p. 291). La publicidad boca a boca se diferencia de otros tipos de mensajes publicitarios debido a que es percibida por los consumidores como una forma fiable de obtener información sobre productos y marcas (Erkan y Evans, 2014).
$\mathrm{La}$ irrupción de Internet ha transformado el estilo de vida de los consumidores y también su forma de relacionarse con las marcas. Percibidas como espacios abiertos y sin limitaciones, muchas plataformas online se han convertido en el principal canal de expresión del boca a boca, haciendo que esta tradicional forma de publicidad interpersonal se integre a la esfera tecnológica (Chiosa, 2014). Las personas comparten sus opiniones y experiencias personales con productos y marcas a través de la red, y esto ha dado surgimiento a un nuevo enfoque para este fenómeno al que se llama boca a boca electrónico o en línea. El boca a boca electrónico [electronic word of mouth $\{\mathrm{eWOM}\}]$ se define como una "declaración hecha por clientes potenciales, reales o anteriores sobre un producto $\mathrm{o}$ empresa, que se pone a disposición de una multitud de personas e instituciones a través de Internet" (Hennig-Thurau et al., 2004, p. 39). En resumen, la comunicación de opiniones sobre productos, marcas $\mathrm{y}$ experiencias ya no se realiza sólo de forma interpersonal cara a cara, sino que hoy está mediada por las TIC.

El boca a boca y el boca a boca en línea son dos conceptos que aunque sean similares en su propósito y ambos tengan una motivación no-comercial, son al mismo tiempo muy diferentes (tabla 1). Numerosos estudios realizados coinciden en que son las formas publicitarias más capaces de influir en el proceso de decisión de compra, ya que se utilizan para obtener información antes, 
durante y después de consumir un producto

o servicio determinado (Huete-Alcocer, 2017).

Los investigadores y los especialistas en marketing y publicidad conocen desde hace tiempo la capacidad de la comunicación boca a boca de influir en las decisiones de compra de los consumidores, pero en la era del eWOM, es necesario profundizar en el análisis de este concepto, sobre todo en lo relacionado con los jóvenes, principales protagonistas del fenómeno.

\section{Tabla 1.}

Diferencias entre el boca a boca y el boca a boca en línea

BOCA A BOCA (WOM)

BOCA A BOCA EN LÍNEA

(eWOM)

\begin{tabular}{|c|c|c|}
\hline Credibilidad & $\begin{array}{l}\text { Quien recibe la información } \\
\text { conoce al comunicador. }\end{array}$ & $\begin{array}{l}\text { Anonimato entre el comunicador y } \\
\text { quien recibe la información. }\end{array}$ \\
\hline Privacidad & $\begin{array}{l}\text { La comunicación es privada, } \\
\text { interpersonal y se lleva a cabo en } \\
\text { tiempo real. }\end{array}$ & $\begin{array}{l}\text { La comunicación compartida no es } \\
\text { privada, y debido a que está escrita } \\
\text { puede ser vista por cualquier } \\
\text { persona en cualquier momento. }\end{array}$ \\
\hline $\begin{array}{l}\text { Velocidad } \\
\text { difusión }\end{array}$ & $\begin{array}{l}\text { Los mensajes se difunden } \\
\text { lentamente. Los receptores } \\
\text { deben estar presentes cuando la } \\
\text { información se comparte. }\end{array}$ & $\begin{array}{l}\text { Los mensajes se difunden } \\
\text { rápidamente entre consumidores y } \\
\text { pueden ser compartidos a través de } \\
\text { Internet en cualquier momento y } \\
\text { lugar. }\end{array}$ \\
\hline Accesibilidad & Menos accesible. & Fácilmente accesible. \\
\hline
\end{tabular}

Nota: información tomada de "A Literature Review of Word of Mouth and Electronic Word of Mouth: Implications for Consumer Behavior", de N. Huete-Alcocer, 2017, Frontiers in Psychology, 8, p. 3 (https://doi.org/10.3389/fpsyg.2017.01256).

\section{Método}

Sobre la base de los conceptos que hemos desarrollado, se diseñó la presente investigación con el objetivo principal de conocer y comprender las variables relacionadas con el consumo y decisiones de compra de los jóvenes argentinos y analizar el rol de las diferentes formas de publicidad en esos procesos. El supuesto subyacente es que la publicidad boca a boca

Global Media Journal México 17(33). julio - diciembre 2020. Pp. 123-138. 
en todas sus formas tiene un rol fundamental en la decisión de compra de los consumidores. En particular, creemos que los jóvenes tienen más confianza en la publicidad boca a boca frente a los otros formatos publicitarios.

\section{Diseño y procedimiento}

Para alcanzar el objetivo, se diseñó un estudio exploratorio con una primera etapa cualitativa y una segunda etapa cuantitativa.

A partir del estudio internacional de la consultora Nielsen (2015) se elaboró un cuestionario que abordaba las principales variables de estudio. Para verificar la validez de este instrumento y para poder establecer las categorías de cada pregunta adaptadas al contexto argentino, se diseñó un primer abordaje cualitativo a partir de la realización de una serie de grupos focales. Es decir, en este caso, la etapa cualitativa fue un paso previo para desarrollar el instrumento de medida cuantitativo (Igartua, 2006).

La preparación de los grupos de discusión se realizó en tres pasos: la programación de la dinámica de grupo con base en los objetivos de la investigación, el diseño de la guía de tópicos o de detonadores, también con base en objetivos y de acuerdo al diseño del cuestionario previo y posteriormente se definieron los criterios de reclutamiento para los participantes. La muestra se escogió mediante una selección de tipo intencional a través de la invitación y reclutamiento de estudiantes de grado y posgrado de dos universidades (una pública y una privada) y de un centro de enseñanza terciaria de oficios, todos comprendidos entre las edades de 18 y 35 años.

Así, los participantes fueron citados en grupos de seis personas, buscando que hubiera representantes de las diferentes variantes de la hoja de control que incluía género, edad, nivel educativo y nivel socioeconómico. Con esta dinámica se buscó obtener una amplia representatividad del universo de estudio. En total se realizaron cinco grupos focales.

La información obtenida a través del análisis de las discusiones de los grupos fue determinante a la hora de elaborar el cuestionario final, que luego fue aplicado en la etapa cuantitativa. Debido a que las preguntas y escalas utilizadas por Nielsen (2015) estaban en idioma inglés, se procedió a traducir las diferentes categorías y opciones a partir de lo expresado por los participantes en las sesiones grupales. En Argentina se utilizan muchos anglicismos, por lo que se decidió optar por muchos de ellos al comprobar que mejoraban la compresión de las preguntas (por ej. review, smartphone, apps, influencer, online, etc.).

Asimismo, a partir de los datos extraídos de los grupos focales, se elaboraron algunas preguntas extras acerca de hábitos de consumo y se optó por limitar a cinco las categorías de productos sobre las cuales se realizarían dichas preguntas: 
tecnología, indumentaria, ocio, vehículos y viajes.

En segundo lugar, la etapa cuantitativa de este trabajo tuvo como objetivo recoger información acerca de la frecuencia y distribución de las variables objeto de estudio a través de un cuestionario. Para la aplicación de este cuestionario se realizó un muestreo por conveniencia, ya que los participantes fueron seleccionados debido a su disponibilidad y porque estaban dentro de la población de interés, pero sin ningún criterio estadístico. Este tipo de muestreo no probabilístico es muy utilizado en la investigación en comunicación, donde lo que se busca es analizar la relación entre variables y establecer inferencias explicativas, no necesariamente dirigidas a realizar una inferencia poblacional $o$ generalizar sus resultados (Igartua, 2006).

En este caso, participaron en el estudio 222 sujetos, estudiantes de diversas carreras de grado y posgrado de una universidad pública y otra privada, y de un centro de enseñanza terciaria de Córdoba, Argentina. La muestra estuvo comprendida por participantes con edades entre 18 y 35 años $(M=24,54)$ y el 67 por ciento fueron mujeres. El trabajo de campo fue realizado durante 2019.

\section{Instrumento y variables}

Para la recogida de datos del estudio cuantitativo se utilizó un cuestionario autoadministrado que recolectó información a partir de la formulación de preguntas cerradas con diferentes opciones.

Confianza en los formatos publicitarios. Se utilizó una escala de cinco puntos donde se solicitaba a los participantes que indicaran el grado de confianza $(1=$ nada, $2=$ poco, $3=\operatorname{algo}, 4=$ bastante, 5 = mucho) para cada uno de los formatos publicitarios. Esta escala es la utilizada por Nielsen (2015) y la traducción de las diferentes opciones se ajustó según los resultados de los grupos focales. A partir de las puntuaciones de la escala, se creó un indicador de grado de confianza.

Motivación para la compra de los diferentes formatos publicitarios. Se utilizó una escala de cinco puntos donde se solicitaba a los participantes que indicaran el grado en que cada formato publicitario los motivaba para la compra $(1=$ nada, $2=$ poco, $3=$ algo, 4 = bastante, $5=$ mucho o). Esta escala fue también utilizada en el estudio global de Nielsen (2015) y se utilizaron las mismas categorías que para la escala anterior. A partir de las puntuaciones obtenidas, se creó un indicador de grado de motivación para la compra.

Elogio y crítica. Se preguntó a los encuestados si alguna vez habían elogiado o criticado públicamente alguna marca, producto o servicio $\mathrm{y}$ a través de qué medios. Las opciones para cada pregunta fueron elaboradas a partir de los resultados de los grupos focales.

Información precisa. Se solicitó a los participantes que estimaran de qué 
modo se puede obtener información precisa sobre un producto/servicio previo a la compra. Las opciones de respuesta fueron elaboradas a partir de los resultados de los grupos focales.

Hábitos de consumo. Se preguntó a cada participante cuál había sido el último producto comprado en estas cinco categorías: tecnología, indumentaria, ocio, vehículos y viajes. Luego para cada uno de ellos se solicitó que indicara: medio en el cual lo había visto por primera vez (diferenciando canales online y offline), si y cómo se había informado acerca del producto previo a la compra y cuál fue el factor decisivo de dicha compra. Las opciones para cada pregunta fueron elaboradas a partir de los resultados de los grupos focales.

\section{Resultados}

\section{Confianza y motivación para la compra}

Los resultados demuestran que la publicidad más creíble proviene directamente de las personas que conocemos y confiamos, pero esta confianza no se limita solo a aquellos del círculo íntimo, sino los participantes declararon que también confian en las opiniones de los consumidores publicadas en línea, convirtiéndolo en el segundo formato más confiable. Los sitios web propios de las marcas se ubican como el tercer formato publicitario más confiable (figura 1).

Por otro lado, es posible apreciar que la proliferación de formatos en línea ha erosionado la confianza en los canales tradicionales. Los encuestados reportaron bajos índices de confianza en los anuncios de televisión, diarios, revistas y radio.

En cuanto a la motivación para la compra, los resultados reflejan que los encuestados que confían en las opiniones de amigos y familiares también reportan que estas opiniones los motivan a llevar a cabo la compra. Por otro lado, el índice de motivación para la compra relativo a las opiniones de consumidores publicadas online es menor que el índice de confianza.

\section{A partir de estos resultados} podemos deducir que la confianza en un formato publicitario es un requisito para que el consumidor se vea motivado a llevar a cabo la acción de compra. Sin embargo, cuando hablamos de publicidad en redes sociales, la motivación para la compra excede el grado de confianza reportado por los encuestados. Lo mismo sucede en relación a los sitios web de las marcas, los cuales se sitúan como el segundo formato más motivador para la compra. 


\section{Figura 1.}

Grado de confianza y motivación para la compra en/de los diferentes formatos publicitarios

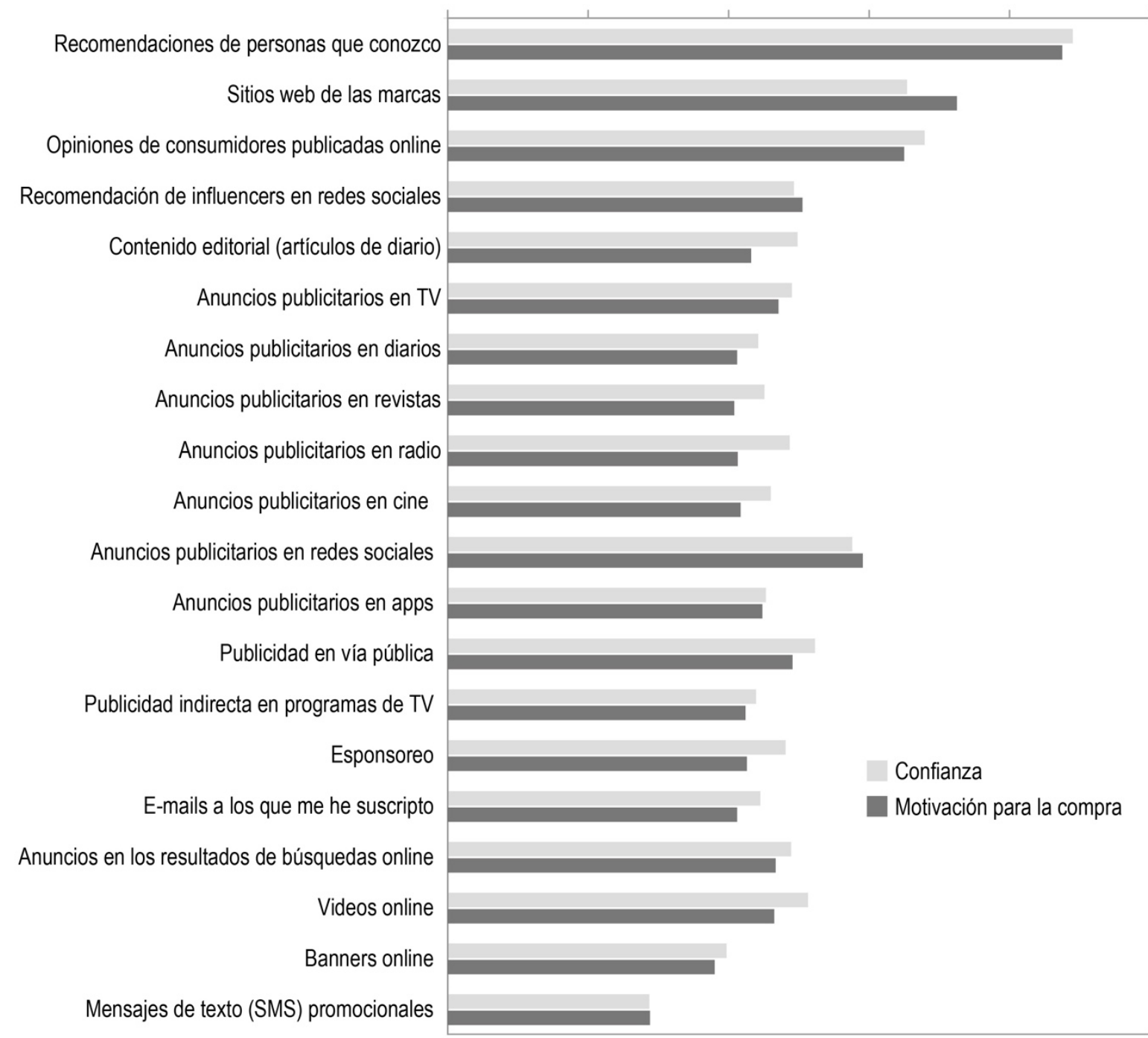

\section{Elogio y crítica}

Los encuestados reconocieron haber criticado públicamente producto o servicio tanto a través de publicaciones o mensajes negativos en las páginas de la propia marca en redes sociales $(14.55 \%)$ como en sus perfiles personales (12.27\%). Asimismo, el $19.09 \%$ de los participantes declaró haber criticado un producto o servicio personalmente frente a otras personas y el
15.91\% efectuó la crítica en la tienda (figura 2).

Cuando se trata de elogiar o expresar satisfacción con un producto o servicio, el $28.18 \%$ afirmó haberlo hecho en persona a otras personas, mientras que el $19.5 \%$ lo hizo alguna vez en la página de redes sociales de la marca y el $20 \%$ a través de publicaciones en perfiles sociales personales (figura 3 ). 


\section{Figura 2.}

Críticas públicas hacia un producto o servicio

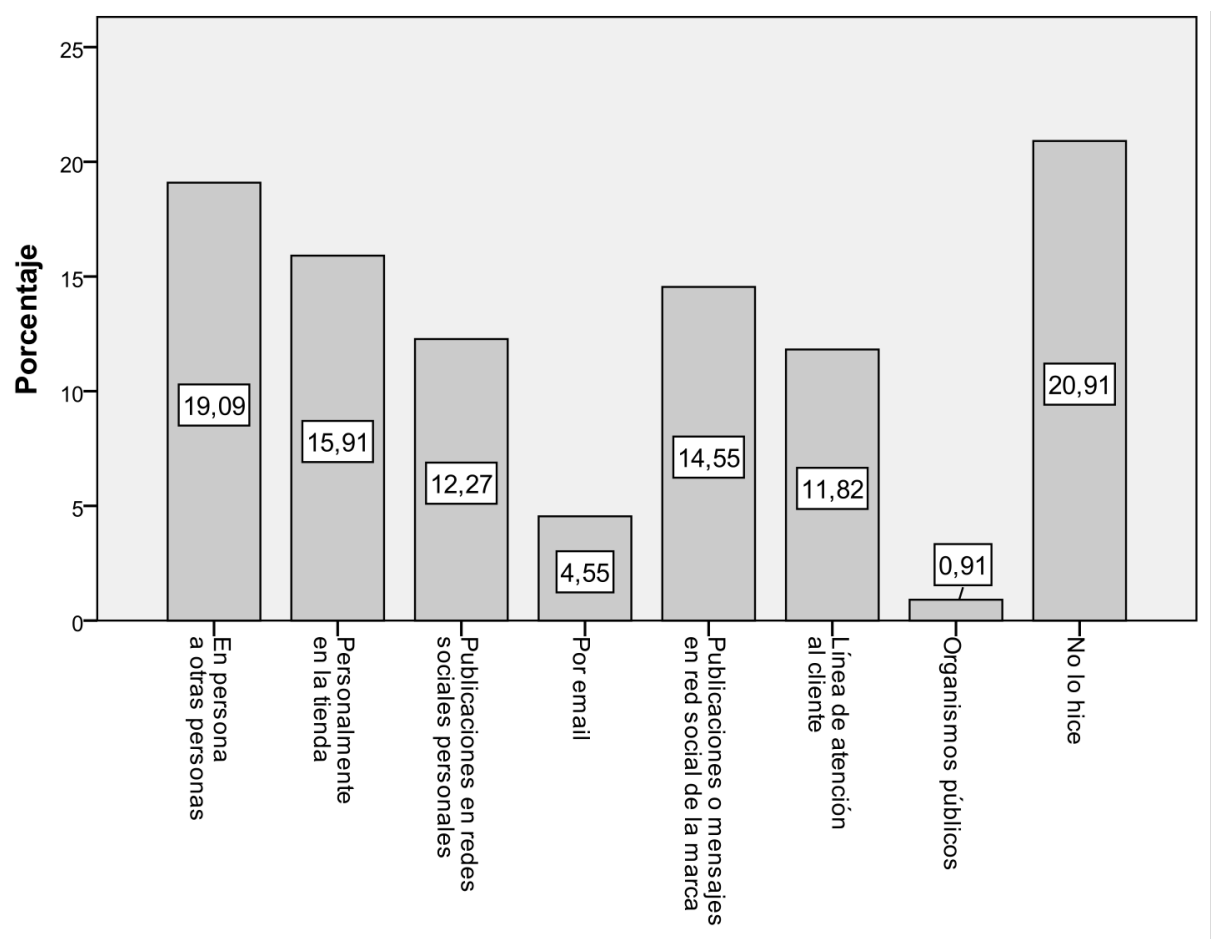

Figura 3.

Elogios públicos hacia un producto o servicio

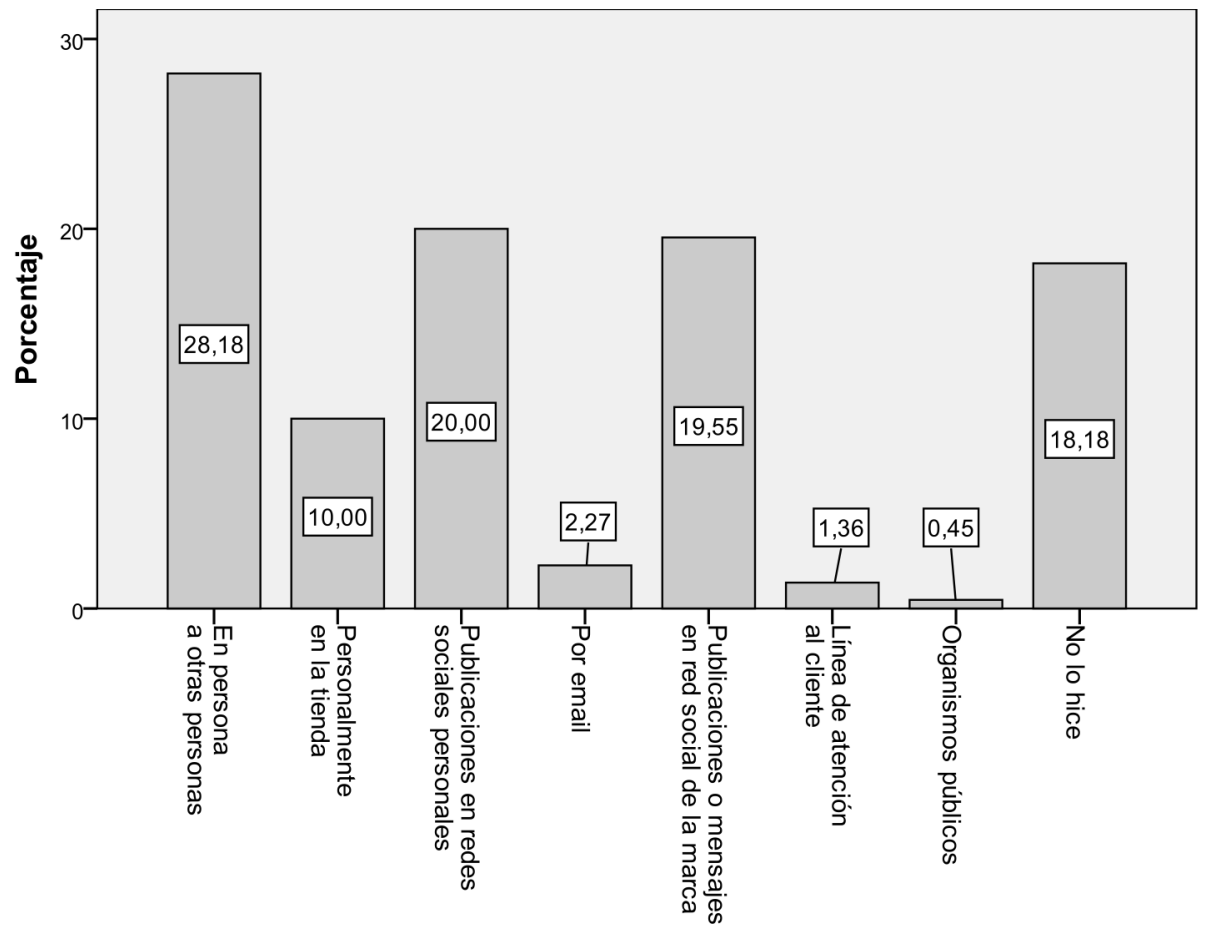

Global Media Journal México 17(33). julio - diciembre 2020. Pp. 123-138. 


\section{Información precisa}

Los participantes expresaron que cuando buscan información precisa sobre un producto o servicio, la gran mayoría (46\%) considera que quien se la aportará son sus amigos, familiares y conocidos, seguidos por las reseñas o reviews online (20.7\%) (figura 4).

\section{Figura 4.}

Información precisa sobre un producto o servicio

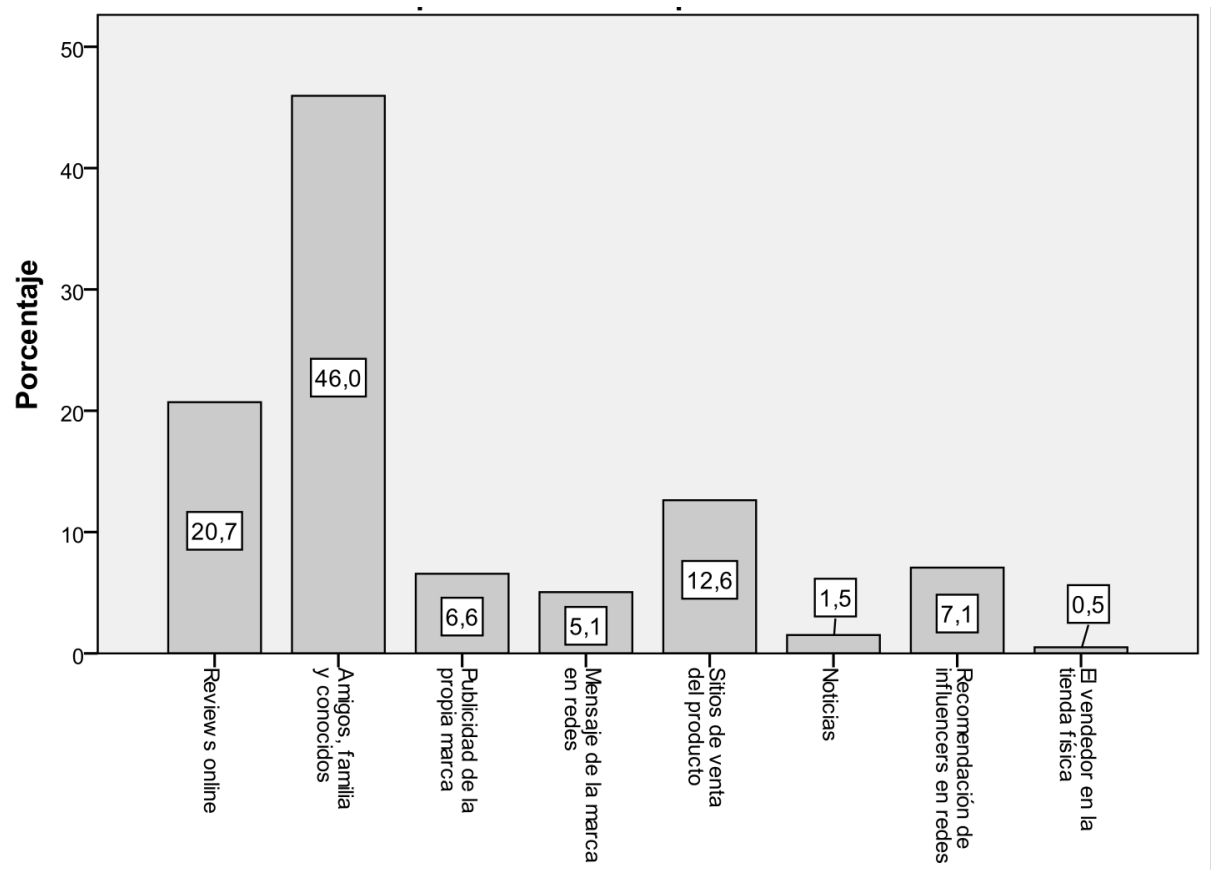

Esto puede verse claramente en los de obtener información previa a la compra resultados relativos a la compra de fue a través de reseñas online de otros tecnología. El 48.3\% de los jóvenes declaró consumidores $(20.2 \%)$, porcentaje que que el último producto que habían iguala a la consulta en persona en la tienda comprado fue un smartphone y que el modo (figura 5). 


\section{Figura 5.}

Búsqueda de información previa a la compra de tecnología

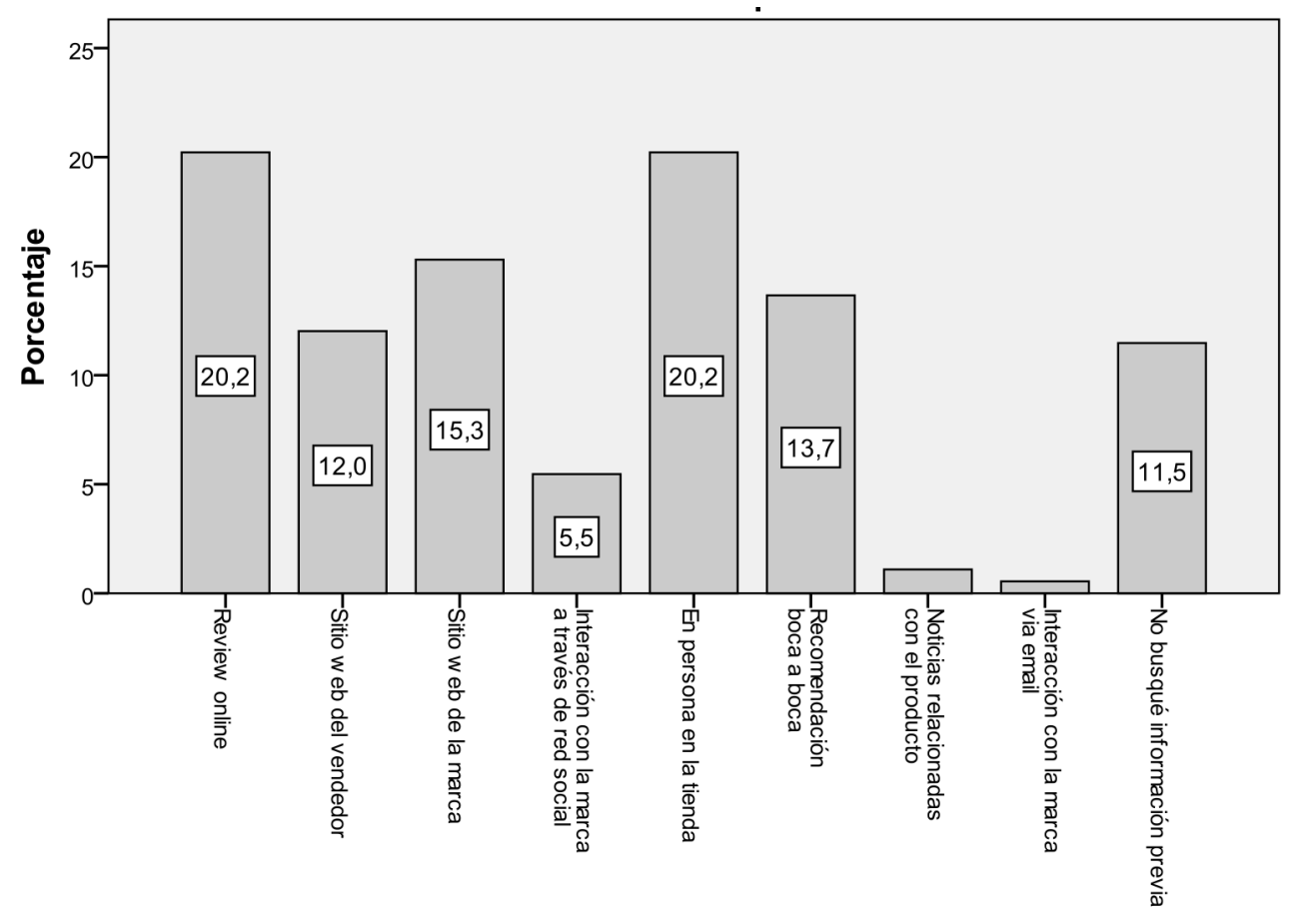

Discusión

Los resultados de este trabajo evidencian que el enfoque de la publicidad clásica se ha vuelto cada vez menos popular entre los consumidores. Podemos asumir que los jóvenes ya no tienen tiempo ni interés para prestar atención a los formatos publicitarios tradicionales y los ignoran cada vez más, mientras que, por el contrario, la comunicación interpersonal gana protagonismo y confían cada vez más en las recomendaciones de su entorno social y de extraños en línea. A su vez, este tipo de publicidad boca a boca es la que más los motiva para la compra. Por último, no hay que perder de vista el hecho de que los sitios web de las marcas están presentes entre los formatos más confiables y motivadores.

Si hacemos la clásica distinción del marketing entre paid media, owned media y earned media (Burcher, 2012), podemos decir que la confianza en los formatos publicitarios tradicionales (medios pagados) desciende a la vez que aumenta en los medios propios, tales como los sitios web. Estos últimos no solo son escaparates de los productos o servicios de una empresa, sino que crean canales específicos de interacción con sus públicos y elaboran mensajes que están bajo el control de la marca. Por otro lado, vemos que las recomendaciones en las que más confían los jóvenes son las enmarcadas en el boca a boca tradicional (WOM) y electrónico (eWOM), 
modalidades que se ubican dentro de los conocidos como medios ganados, ya que son mensajes y contenidos que circulan sobre la marca, sin que se haya pagado por ello.

Los resultados relativos a los anuncios en redes sociales, donde el índice de motivación para la compra es mayor que el índice de confianza, concuerdan con lo reportado en encuestas globales como las de la consultora Nielsen. Sus expertos afirman que este tipo de formatos publicitarios donde la intención de compra excede la confianza que genera el anuncio suelen tener un atributo particular: el fácil acceso al producto o servicio. Es decir, este tipo de publicidad online hace que sea muy fácil para el consumidor llevar a cabo alguna acción en relación al anuncio, aunque el anuncio no le haya generado mucha confianza. Esto es debido a que con un simple clic puede dirigirse al sitio donde buscar más información o directamente aprovechar la oportunidad de comprar el producto o servicio (Nielsen, 2015).

También hemos podido observar que antes de realizar una compra, especialmente si se trata de un producto nuevo o algo caro, a los consumidores les gusta investigar un poco de antemano. En este caso, las personas no sólo acuden a la opinión de los 'expertos' en las tiendas sino que también buscan qué es lo que otros consumidores han dicho online. Esto hace que uno de los formatos protagonistas en el boca a boca electrónico sean las reseñas en línea. Algunos autores consideran que la confianza en este tipo de mensajes se debe a que son percibidos como creados por personas que no tienen un interés propio en promocionar el producto o servicio, y por lo tanto son más creíbles (Kundu y Sundara Rajan, 2017).

No hay dudas de que el advenimiento de la tecnología y el auge de las redes sociales han cambiado la forma en que los consumidores perciben los mensajes publicitarios. Los procesos de toma de decisión de compra se han transformado y la publicidad tradicional empieza a perder su efecto frente a las formas de publicidad boca a boca (Subramanian, 2018). El nuevo consumidor, está conectado y comparte experiencias e información ilimitada, de manera que todas esas experiencias de consumo, sean físicas o virtuales, pesan de manera contundente en la recomendación del producto o servicio (Aguirre Valencia, 2016). Esto no se limita a las opiniones negativas que reflejan la insatisfacción con algún producto o marca, sino que la satisfacción también se propaga y si el consumidor está complacido con algo que ha comprado o contratado, también lo compartirá (Castelló Martínez, 2016).

Asimismo, el auge de la modalidad de compra online, hace que cuando los jóvenes consumidores reciban sugerencias a través del boca a boca electrónico, puedan visitar en el mismo momento el sitio web o tienda y adquirir ese producto o servicio. En otras palabras, si un consumidor toma en consideración una recomendación, esta puede convertirse en una acción de compra 
al instante, y esa es una de las características destacadas que hacen que el boca a boca online sea tan importante (Erkan y Evans, 2014).

Las empresas y marcas deben comprender la influencia de este tipo de publicidad sobre las decisiones de compra de un producto o servicio. Sabemos que las personas definitivamente están influenciadas por miembros de la familia, amigos y conocidos en quienes confían, pero aquí se demuestra que también confían en los consejos de completos desconocidos a través de sus opiniones en línea. Esto también se aplica a cualquier consejo previo a la compra e información sobre los productos que se obtiene a través de dichas interacciones.

\section{Conclusiones}

Los resultados demuestran que las comunicaciones boca a boca y boca a boca electrónico tienen un rol fundamental en la decisión de compra de los jóvenes consumidores. Es decir, es más probable que los consumidores confíen en los consejos y opiniones de sus pares (aunque sean personas desconocidas) que en los anuncios que provienen directamente de la marca a través de la publicidad tradicional (Subramanian, 2018).

La publicidad moderna ya no se puede concebir sin tener en cuenta las recomendaciones boca a boca electrónico, que hoy ya son un factor decisivo en el éxito de una marca (Meiners et al., 2010). En este sentido, creemos que el reto para las empresas y marcas es entablar una relación con el consumidor basada en la confianza, autenticidad y transparencia a través de sus acciones de comunicación.

Asimismo, los anunciantes pueden obtener un gran provecho de esta nueva realidad, ya que cuando los jóvenes consumidores expresan en línea sus experiencias, opiniones, críticas, valoraciones o sugerencias de mejora sobre los productos y servicios, ponen a disposición de las marcas información muy valiosa que antes solo se podía obtener a través de costosas herramientas tales como las encuestas o grupos de discusión (MuelaMolina y Pazos, 2010).

El hecho de que el boca a boca sea un factor importante en los procesos de decisión de compra es una de las razones por las cuales las marcas deben adaptar sus estrategias publicitarias y escuchar lo que se dice sobre ellas, su mercado y sus competidores, necesitan aprender de lo que 'escuchan' y también responder cuando sea necesario. Según Nielsen (2015), la mejor recomendación para las marcas es seguir evolucionando y reemplazar los argumentos de venta unidireccionales por una comunicación activa bidireccional, transparente y responsable, es decir, unirse a la conversación.

Con respecto a las limitaciones de este estudio, debemos señalar la falta de representatividad del tipo de muestreo 
empleado y la imposibilidad de hacer aseveraciones estadísticas sobre los resultados, así como el riesgo de haber incurrido en sesgos debido al criterio de selección utilizado.

Por otro lado, estimamos que este trabajo es un aporte a los estudios sobre la comunicación boca a boca, así como para los análisis sobre toma de decisiones de compra de los jóvenes latinoamericanos. Creemos que sería interesante estudiar la temática en el contexto pandemia y pospandemia, donde las interacciones de los jóvenes en casi todos los ámbitos de la vida cotidiana están siendo mediadas por las TIC.

Asimismo, sería interesante analizar la comunicación boca a boca no solo en relación a actitudes frente a la publicidad sino incorporando conceptos relacionados con las actitudes hacia la marca, tales como la lealtad de marca o el compromiso del consumidor.

\section{Referencias bibliográficas}

Aguirre Valencia, V. (2016). Las redes. El valor de la experiencia colectiva. En I. ZacipaInfante, V. Tur-Viñes y J. Segarra-Saavedra (Coords.), Tendencias publicitarias en Iberoamérica. Diálogo de saberes y experiencias (pp. 339-351). Universidad de Alicante.

AIMC (Asociación para la Investigación de Medios de Comunicación). (30 de noviembre de 2010). Internet, en medio de los medios. Asociación para la Investigación de Medios de Comunicación. https://www.aimc.es/a1mcc0nt3nt/uploads/2010/11/Internet_medio_medios.pdf

Arndt, J. (1967). Role of product related conversations in the diffusion of a new product. Journal of Marketing Research, 4, 291-295.

Benavides Delgado, J. (2012). La investigación en comunicación y publicidad: Nuevos temas y problemas. Questiones Publicitarias, I(17), 71-93.

Burcher, N. (2012). Paid, owned, earned: Maximising marketing returns in a socially connected world. Kogan Page.

Castelló Martínez, A. (2016). El marketing de influencia. Un caso práctico. En I. ZacipaInfante, V.Tur-Viñes y J. Segarra-Saavedra (Coords.), Tendencias publicitarias en Iberoamérica. Diálogo de saberes y experiencias (pp. 49-65). Universidad de Alicante.

Chiosa, A. (2014). Word of mouth on social media. SEA - Practical Application of Science, II(4), 37-42.

Costa, J. (2007). Pospublicidad. La era de la comunicación global. Pensar la publicidad, l(1), 41-54. 
Erkan, I., y Evans, C. (2014, junio). The impacts of electronic word of mouth in social media on consumers' purchase intentions [presentación de artículo]. International Conference on Digital Marketing [Conferencia]. Colombo, Sri Lanka.

Geraci, J., y Nagy, J. (2004). Millennials - The new media generation. Young Consumers: Insight and Ideas for Responsible Marketers, 5(2), 17-24.

Hennig-Thurau, T., Gwinner, K., Walsh, G., y Gremler, D. (2004). Electronic word-of mouth via consumer-opinion platforms: what motivates consumers to articulate themselves on the internet? Journal Interactive Marketing, 18(1), 38-52.

Howe, N., y Strauss, W. (2000). Millennials rising: The next great generation. Vintage Books.

Huete-Alcocer, N. (2017). A literature review of word of mouth and electronic word of mouth: implications for consumer behavior. Frontiers in Psychology, 8, 1-4.

Igartua, J. (2006). Métodos cuantitativos de investigación en comunicación. Bosch.

Kundu, S., y Sundara Rajan, C. (2017). Word of mouth: A literature review. International Journal of Economics \& Management Sciences, 6(6).

Meiners, N., Schwarting, U., y Seeberger, B. (2010). The renaissance of word-of-mouth marketing: A 'new' standard in twenty-first century marketing management. International Journal of Economic Sciences and Applied Research, 3(2), 79-97.

Mejia-Giraldo, J. (2016). La ilusión virtual de poder del consumidor en las sociedades del conocimiento posindustriales. En I. Zacipa-Infante, V.Tur-Viñes y J. Segarra-Saavedra (Coords.), Tendencias publicitarias en Iberoamérica. Diálogo de saberes y experiencias (pp. 185-200). Universidad de Alicante.

Muela-Molina, C., y Pazos, A. (2010). Jóvenes y publicidad on line: Nuevos espacios y formas, otros retos. Revista de Estudios de Juventud, 88, 183-199.

Nielsen (2015). Global trust in advertising. Winning strategies for an evolving media landscape. $\quad 2015 . \quad$ September 2 https:/www.nielsen.com/wpcontent/uploads/sites/3/2019/04/global-trust-in-advertising-report-sept-2015-1.pdf

Sánchez, L., Megías, I., y Rodríguez, E. (2004). Jóvenes y publicidad. Valores en la comunicación publicitaria para jóvenes. INJUVE y FAD.

Subramanian, K. (2018). Social media and the word of mouth publicity. International Research Journal of Advanced Engineering and Science, 3(2), 95-100.

Tur-Viñes, V. (2016). Publicidad, tendencias de futuro y Educación Superior. En I. ZacipaInfante, V.Tur-Viñes y J. Segarra-Saavedra (Coords.), Tendencias publicitarias en Iberoamérica. Diálogo de saberes y experiencias (pp. 1-26). Universidad de Alicante.

Vivas López, M. (2007). Comunicación pública y formación del espacio público político. Folios, 10(12-13), 8-16. 